\title{
Multiple coexisting intercalation structures of sodium in epitaxial graphene-SiC interfaces
}

\author{
Andreas Sandin, ${ }^{1}$ Thushari Jayasekera, ${ }^{2}$ J. E. Rowe, ${ }^{1}$ Ki Wook Kim, ${ }^{3}$ M. Buongiorno Nardelli,,${ }^{1,4,}{ }^{*}$ and Daniel B. Dougherty ${ }^{1, \dagger}$ \\ ${ }^{1}$ Department of Physics, North Carolina State University, Raleigh, North Carolina, 27695-8202, USA \\ ${ }^{2}$ Department of Physics, Southern Illinois University-Carbondale, Carbondale, Illinois, 62901-4401, USA \\ ${ }^{3}$ Department of Electrical and Computer Engineering, North Carolina State University, Raleigh, North Carolina 27695-7911, USA \\ ${ }^{4}$ Computer Science and Mathematics Division, Oak Ridge National Laboratory, Oak Ridge, Tennessee 37831-6359, USA
}

(Received 23 January 2012; published 9 March 2012)

\begin{abstract}
We show using scanning tunneling microscopy, spectroscopy, and ab initio calculations that two intercalation structures coexist for $\mathrm{Na}$ in epitaxial graphene on $\mathrm{SiC}(0001)$. Intercalation takes place at room temperature, and $\mathrm{Na}$ electron dopes the graphene. It inserts in between single-layer graphene and the interfacial layer and also penetrates beneath the interfacial layer and decouples it to form a second graphene layer. Decoupling is accelerated by annealing and is verified by $\mathrm{Na}$ deposition onto the interface layer combined with computational modeling of the two new decoupled buffer layer structures.
\end{abstract}

DOI: 10.1103/PhysRevB.85.125410

PACS number(s): 73.22.Pr, 61.48.Gh, 68.37.Ef, 74.20.Pq

\section{INTRODUCTION}

Graphene is the most promising electronic material to be discovered in the past decade. ${ }^{1}$ Its high carrier mobility and chemical and mechanical robustness suggest important applications in electronics that have already started to be realized in prototype devices. ${ }^{2}$ A crucial goal in ongoing graphene research is to find ways to control its physical properties by chemical doping.

One strategy for controlling the physical properties of graphene has been adapted from the field of graphite intercalation compounds. ${ }^{3}$ It is possible to insert impurity atoms in between sheets of graphite, where they become chemically bound. The most striking example is calcium intercalation in graphite, which results in a superconducting solid $\left(\mathrm{CaC}_{6}\right)$ with a transition temperature of above $11 \mathrm{~K}^{4}$ Unfortunately, intercalation processes in graphite are often inhibited by slow kinetics. ${ }^{3,5}$ Intercalation beneath graphene may be more efficient because intercalating species have only to be inserted beneath a single layer or perhaps a few atomic layers.

Several atomic species have been intercalated beneath epitaxial graphene grown on both $\mathrm{SiC}(0001)$ and metallic substrates. On the Si-terminated $\mathrm{SiC}(0001)$ surface, graphene is allowed to grow nearly decoupled from substrate supported by an interfacial carbon-rich layer (also known as "buffer layer") that can be seen as a covalently bonded graphenelike sheet ${ }^{6}$ with a large band gap and vanished Dirac cone band structure. $^{7}$ Recent studies ${ }^{8-10}$ demonstrated that this buffer layer could be "activated" by intercalation of hydrogen atoms at high temperatures. Hydrogen intercalation breaks covalent bonds between the buffer layer and the $\mathrm{SiC}$ substrate to transform this interfacial layer into a purely $\mathrm{sp}^{2}$-bonded sheet of graphene.

Other experimental studies have demonstrated intercalation structures for oxygen ${ }^{11}$ and for compounds that can alternate the carrier concentration in the graphene. Fluorine intercalation gives $\mathrm{p}$-doped graphene $^{12}$ versus $\mathrm{n}$-doped graphene from alkali metal (Li) intercalation. ${ }^{13,14}$ Gold intercalation beneath single-layer graphene (SLG) on $\mathrm{SiC}(0001)$ was reported to $\mathrm{p}$ dope the graphene layer. ${ }^{15,16}$ Theoretical studies demonstrated that modifications in the chemical composition of the buffer can lead to significant changes of the graphene bands, allowing for a fine tuning of the electronic structure of the system with band offsets up to $1.5 \mathrm{eV}$ and even inducing magnetism of the graphene. ${ }^{17}$

Alkali metal adsorption on graphene has been used extensively to study the effects of electron doping. Potassium deposition on graphene on $\mathrm{SiC}(0001)$ has enabled detailed studies of quasiparticles ${ }^{18}$ and the discovery of plasmarons ${ }^{19}$ in graphene. The additional dipole field from adsorbed potassium can also act to break the sublattice symmetry in graphene to induce the band-gap opening. ${ }^{20}$ Doping induced by intercalation in graphene may be more significant and could enable studies of correlated electron phenomena such as superconductivity or ferromagnetism in graphene because of the presence of extended Van Hove singularities. ${ }^{21}$ The intercalation of $\mathrm{Ca}$ was reported by McChesney et al. to result in electron doping of epitaxial graphene on $\mathrm{SiC}(0001)$ that moved the Dirac point an additional $\sim 0.5 \mathrm{eV}$ below the Fermi level. ${ }^{21}$

The most prominent studies of doping induced by intercalation have been carried out using angle-resolved photoemission spectroscopy (ARPES). Because this technique samples a macroscopic area of the sample, it may average over spatially inhomogeneous structures with different electronic properties. The purpose of this paper is to report the coexistence of two distinct intercalation structures of $\mathrm{Na}$ at interfaces in the epitaxial graphene on the $\mathrm{SiC}(0001)$ system. We describe scanning tunneling microscope (STM) studies of the intercalation of sodium in epitaxial graphene on $\mathrm{SiC}(0001)$ at room temperature and the effects of subsequent annealing on the intercalation structures. Sodium atoms form complex spatially inhomogeneous structures that include intercalation directly underneath SLG and intercalation beneath the carbonrich interfacial "buffer layer." The location of $\mathrm{Na}$ in these intercalation structures and its impact on electronic structure (in particular, buffer layer decoupling ${ }^{8}$ ) has been modeled with $a b$ initio density functional theory (DFT) calculations.

Electron-doping effects in each new intercalation structure are inferred from the scanning tunneling spectroscopy (STS) study of image-potential-derived surface states (IPS's). These states arise outside of a surface due to polarization of surface charge ${ }^{22}$ and have recently been connected with the 
formation of interlayer states in graphite $^{23,24}$ and used to understand screening and doping effects in epitaxial graphene in $\mathrm{SiC}(0001) .{ }^{25}$ Changes in work function because of doping are often visible as corresponding shifts in the energy of IPS's ${ }^{26}$ and demonstrate doping effects in sodium-intercalated epitaxial graphene. Most importantly, STS results have been connected with work-function changes calculated from first principles that unambiguously distinguish the different locations of $\mathrm{Na}$ in the intercalation structures.

\section{EXPERIMENTAL METHODS}

The SiC samples used in this study were chemicalmechanical polished (NovaSiC) $4 \mathrm{H}-\mathrm{SiC}(0001)$ wafers. They were cleaned ex situ with acetone, methanol, and hydrofluoric acid before introduction to an ultrahigh vacuum (UHV) system (Omicron). Using direct current heating, the samples were annealed in UHV (base pressure $\sim 2 \times 10^{-11}$ Torr) to $1300^{\circ} \mathrm{C}$ for $3 \mathrm{~min}$ to grow graphene. Surfaces prepared in this way typically show primarily SLG with small domains ( 10-20\% surface coverage) of bilayer graphene (BLG). Different layers can be easily distinguished by the reduction in substrateinduced corrugation in the bilayer and by the systematic energy differences in IPS's in STS. ${ }^{25}$

By choosing a lower annealing temperature $\left(1000{ }^{\circ} \mathrm{C}\right.$ for $10 \mathrm{~min}$ ) of the $\mathrm{SiC}$ substrate, we can prepare a surface that consists of mainly a bare buffer layer coexisting with small domains of SLG as confirmed by STM imaging, low-energy electron diffraction, and analysis of the shape of C KLL Auger peaks.

The samples were allowed to cool for several hours to approximately room temperature. Sodium was then deposited from an outgassed getter source (SAES Getters). STM images were measured using a commercial instrument (Omicron) in constant current mode at room temperature with electrochemically etched tungsten tips. Annealing experiments after sodium deposition were carried out using a calibrated tungsten heater in the sample manipulator and are expected to be accurate to about $20^{\circ} \mathrm{C}$. STS was performed in distance versus voltage ["z(V)"] mode with constant current feedback engaged. The junction voltage is swept in a positive large bias interval where high local density of states is observed as an increased rate of tip retraction. ${ }^{27}$ This measurement is the most useful form of STS for studying high-energy IPS's, where normal STS in constant height mode leads to high currents and tip instability.

\section{COMPUTATIONAL METHODS}

Theoretical calculations were carried out using planewave, pseudopotential, DFT as implemented in the quantumESPRESSO software package ${ }^{28}$ with the generalized gradient approximation (GGA) and the Perdew-Becke-Ernzerhof functional and a correction for dispersion forces [DFT(D)]. Other computational details are the same as in Ref. 17. All calculations include $1 \mathrm{Na}$ atom per 8 graphene carbon atoms in a computational unit cell that includes a carbon-rich buffer layer strongly bonded to the $\mathrm{SiC}$ surface but leaving several unsaturated dangling bonds. ${ }^{17}$

\section{RESULTS AND DISCUSSION}

At low coverages, sodium exposure on SLG results in the appearance of linear chains on the surface following the threefold symmetric directions of the $\mathrm{SiC}$ substrate as shown in Fig. 1(a). In Fig. 1(b), at higher submonolayer coverage, the chains become more densely packed and coalesce into islands, which have the same $6 \times 6$ corrugation as the bare SLG [Fig. 1(c)]. The STM images in Fig. 1(d) clearly show these domains to be covered with a honeycomb graphene lattice. We infer that sodium is intercalated in between the buffer layer and the graphene layer. We abbreviate the compact domain in Fig. 1(c) as $\mathrm{SiC} / \mathrm{B} / \mathrm{Na} / \mathrm{G}$, where $\mathrm{B}$ stands for buffer layer and $\mathrm{G}$ for graphene layer. Judging from the apparent height and continuity of this intercalation structure, the islands are locally the same (e.g., SiC/B/Na/G) as the chains. A recent DFT study supports the idea that $\mathrm{Na}$ intercalation between the buffer layer graphene is preferred over $\mathrm{Na}$ adsorption on top of the graphene layer. ${ }^{29}$ Sodium atoms completely avoid domains of BLG [marked in Fig. 1(b)]. Our experimental observation of intercalation in epitaxial SLG is unexpected because $\mathrm{Na}$ does not form intercalation compounds on graphite ${ }^{3}$ without help from catalytic impurites. ${ }^{30}$ The detailed mechanism of this process is a significant open question.

An additional intercalation structure coexists with $\mathrm{SiC} / \mathrm{B} / \mathrm{Na} / \mathrm{G}$ after exposing epitaxial graphene to $\mathrm{Na}$ as illustrated in Figs. 2(a)-2(c). It exists in small domains immediately after deposition of $\mathrm{Na}$ at room temperature but dominates the surface by heating of the sample after $\mathrm{Na}$ deposition. Figure 2(a) shows an area of a sample that has been annealed to approximately $180^{\circ} \mathrm{C}$ that is now strikingly different from the $\mathrm{SiC} / \mathrm{B} / \mathrm{Na} / \mathrm{G}$ layers in Fig. 1. It exhibits a flat morphology with no sign of periodic $6 \times 6$ corrugation from the substrate. Instead, domains have randomly distributed depressions and
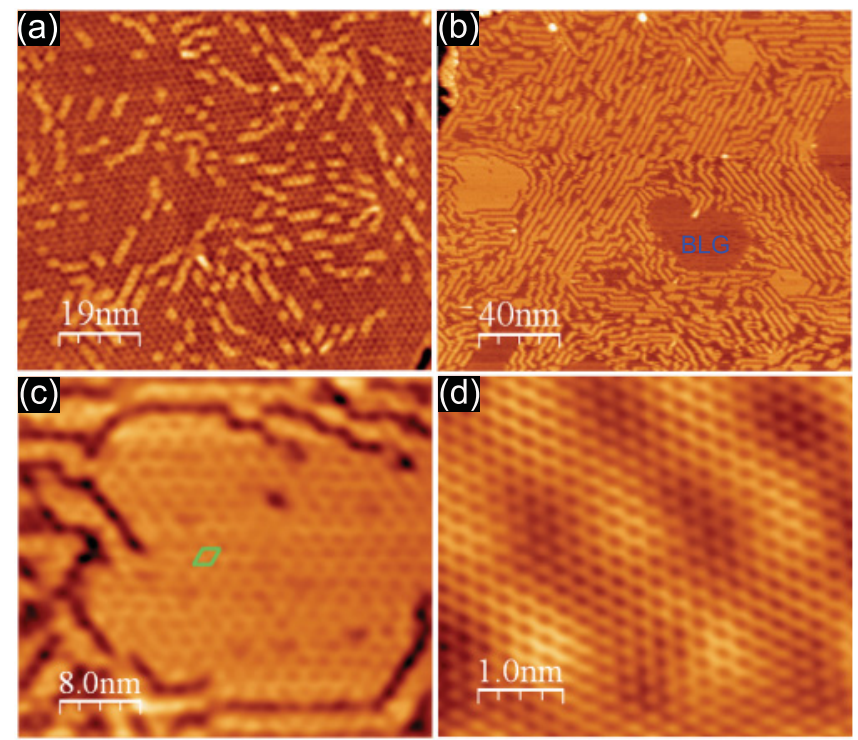

FIG. 1. (Color online) STM images of Na intercalated between buffer and graphene layer, $\mathrm{SiC} / \mathrm{B} / \mathrm{Na} / \mathrm{G}$. (a) Low-coverage Na chains $(\mathrm{V}=-2.18 \mathrm{~V}, 62 \mathrm{pA})$. (b) Intermediate coverage with chains and islands. $(\mathrm{V}=-2.0 \mathrm{~V}, \mathrm{I}=50 \mathrm{pA})$. (c) $\mathrm{Na}$ island in (b) with $(6 \times 6)$ quasicell indicated $(\mathrm{V}=-2.3 \mathrm{~V}, \mathrm{I}=50 \mathrm{pA})$. (d) Graphene lattice of island in $(\mathrm{c})(\mathrm{V}=-0.55 \mathrm{~V}, \mathrm{I}=350 \mathrm{pA})$. 


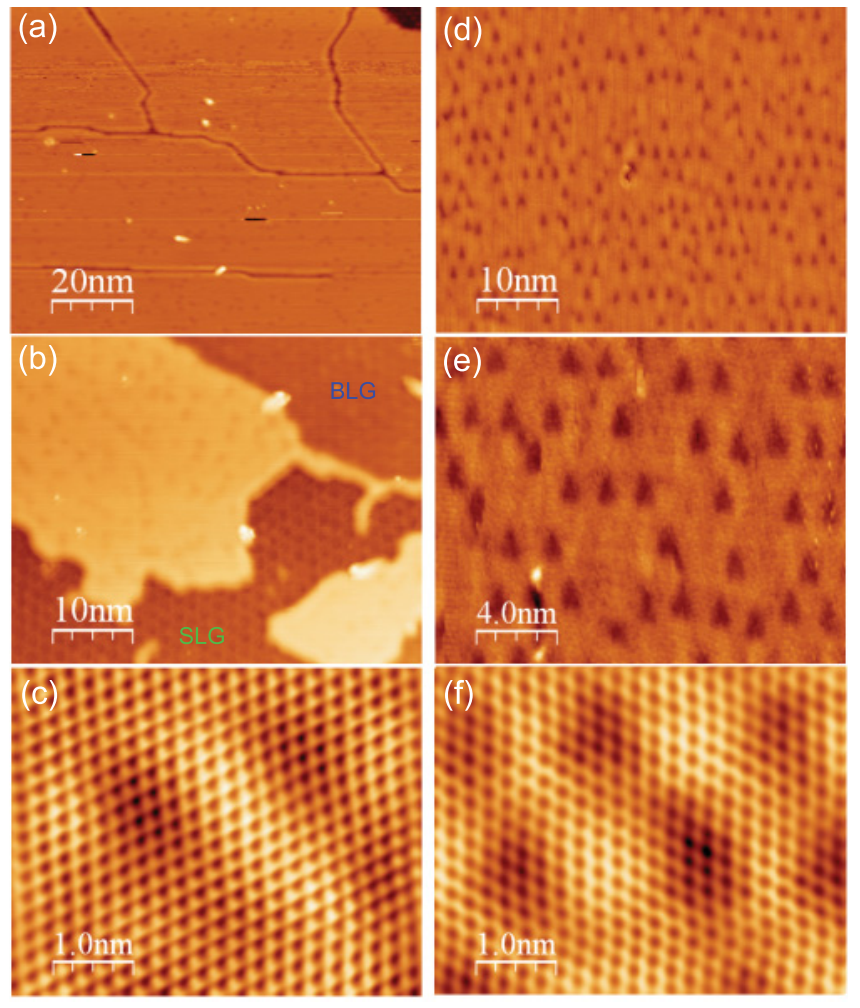

FIG. 2. (Color online) STM images of $\mathrm{Na}$ intercalated at $\mathrm{SiC}$ interface after heating of sample: $\mathrm{SiC} / \mathrm{Na} / \mathrm{G} / \mathrm{G}$ (a)-(c) and $\mathrm{SiC} / \mathrm{Na} / \mathrm{G}$ (d)-(f). (a) $\sim 180{ }^{\circ} \mathrm{C}$ with full-coverage $\mathrm{SiC} / \mathrm{Na} / \mathrm{G} / \mathrm{G}(\mathrm{V}=-1.93 \mathrm{~V}$, $\mathrm{I}=200 \mathrm{pA}$ ). (b) $\sim 530^{\circ} \mathrm{C}$ showing an area of coexisting SLG, BLG, and $\mathrm{SiC} / \mathrm{Na} / \mathrm{G} / \mathrm{G}(\mathrm{V}=-1.7 \mathrm{~V}, \mathrm{I}=50 \mathrm{pA})$. (c) Graphene lattice on $\mathrm{SiC} / \mathrm{Na} / \mathrm{G} / \mathrm{G}(\mathrm{V}=-0.37 \mathrm{~V}, \mathrm{I}=150 \mathrm{pA})$. (d) $\sim 180^{\circ} \mathrm{C}$ with full-coverage $\mathrm{SiC} / \mathrm{Na} / \mathrm{G}(\mathrm{V}=-2.6 \mathrm{~V}, \mathrm{I}=50 \mathrm{pA})$. (e) Triangular depressions covering $\mathrm{SiC} / \mathrm{Na} / \mathrm{G}$ surface $(\mathrm{V}=-1.8 \mathrm{~V}, \mathrm{I}=50 \mathrm{pA})$. (f) Graphene lattice on $\mathrm{SiC} / \mathrm{Na} / \mathrm{G}(\mathrm{V}=-0.14 \mathrm{~V}, \mathrm{I}=0.11 \mathrm{nA})$.

line defects. Figure 2(b) directly demonstrates the comparison between this surface and bare SLG and BLG.

The vanishing corrugation from the substrate is reminiscent of recent STM observations of hydrogen-exposed graphene on $\mathrm{SiC}$, where the hydrogen has intercalated at the $\mathrm{SiC}$ buffer layer, decoupling the buffer layer to create a second graphene layer. ${ }^{10}$ Figure 2 (c) shows a close up of an intercalated domain after annealing of the sample, where it is apparent that the surface is covered graphene but now with a triangular symmetry of the graphene honeycomb. This is strong evidence that we now have two layers of graphene sheets that are stacked in regular Bernal stacking (AB). We, therefore, conclude that the sodium has penetrated beneath the buffer layer and converted it into a second graphene layer just like the case for lithium, ${ }^{13,14}$ hydrogen ${ }^{8-10}$ oxygen, ${ }^{11}$ and fluorine ${ }^{12}$ intercalation. We abbreviate this intercalation structure $\mathrm{SiC} / \mathrm{Na} / \mathrm{G} / \mathrm{G}$.

To directly verify that the buffer layer can convert to a second graphene layer because of $\mathrm{Na}$ intercalation, we repeated the measurement with a surface prepared to consist of bare buffer layer. Images in Figs. 2(d)-2(f) show such a surface after $\mathrm{Na}$ deposition and annealing to $\sim 180^{\circ} \mathrm{C}$. The sodium has intercalated at the buffer layer-SiC interface across the whole surface. We label this geometry as $\mathrm{SiC} / \mathrm{Na} / \mathrm{G}$. Its surface morphology is similar to the previously described structure $\mathrm{SiC} / \mathrm{Na} / \mathrm{G} / \mathrm{G}$, where the $6 \times 6$ surface corrugation has vanished. The STM image in Fig. 2(e) proves that the buffer layer is decoupled because the graphene honeycomb lattice is clearly visible.

To further characterize the three intercalated structures, we measured their IPS's using STS and compared them to a fixed energy reference on the $n=1$ IPS of SLG as described below. Our expectation is that the variation in energy of these states between different intercalation structures will reflect variations in the Na-doping-induced changes in work function compared to SLG. ${ }^{26}$ Figure 3 displays differentiated distance-voltage $(\mathrm{dz} / \mathrm{dV})$ spectra for intercalated structures relative to SLG (red curve, with single $n=1$ IPS peak defined as $0 \mathrm{eV}$ ), where the peak corresponds to the first member of the (odd) ${ }^{24}$ Rydberg series that we refer to as $n=1$.

Because the $n=1$ peak position of epitaxial graphene is sensitive to different tip states due to poor screening in the graphene sheet, ${ }^{25}$ it is important to keep track of the tip state. We collected the STS data for intercalation structures $\mathrm{SiC} / \mathrm{Na} / \mathrm{G}, \mathrm{SiC} / \mathrm{Na} / \mathrm{G} / \mathrm{G}$ and SLG simultaneously (i.e., with the same tip state) for use as an internal reference (in contrast to usual STS studies in which the substrate Fermi level is the most useful reference). The intercalation structure $\mathrm{SiC} / \mathrm{B} / \mathrm{Na} / \mathrm{G}$ was measured simultaneously with BLG and referenced to SLG using the known $0.14 \pm 0.02 \mathrm{eV}$ difference in IPS energy between these two. ${ }^{25}$

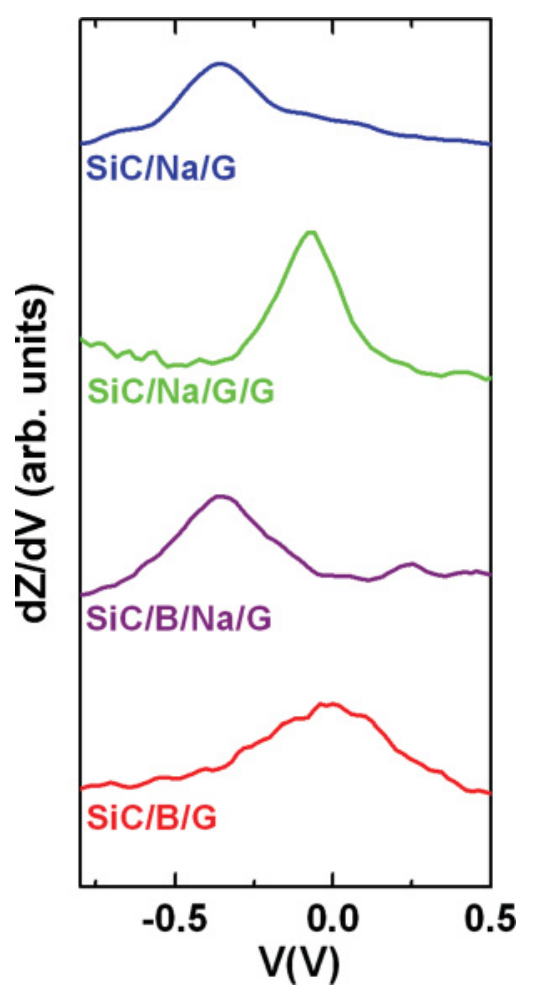

FIG. 3. (Color online) Differentiated (dz/dV) distance-voltage spectra $\mathrm{z}(\mathrm{V})$ of the $n=1$ IPS for different intercalation structures relative to the $n=1$ IPS on SLG (red/dark gray). All spectra are recorded with a constant current set point of $50 \mathrm{pA}$. 

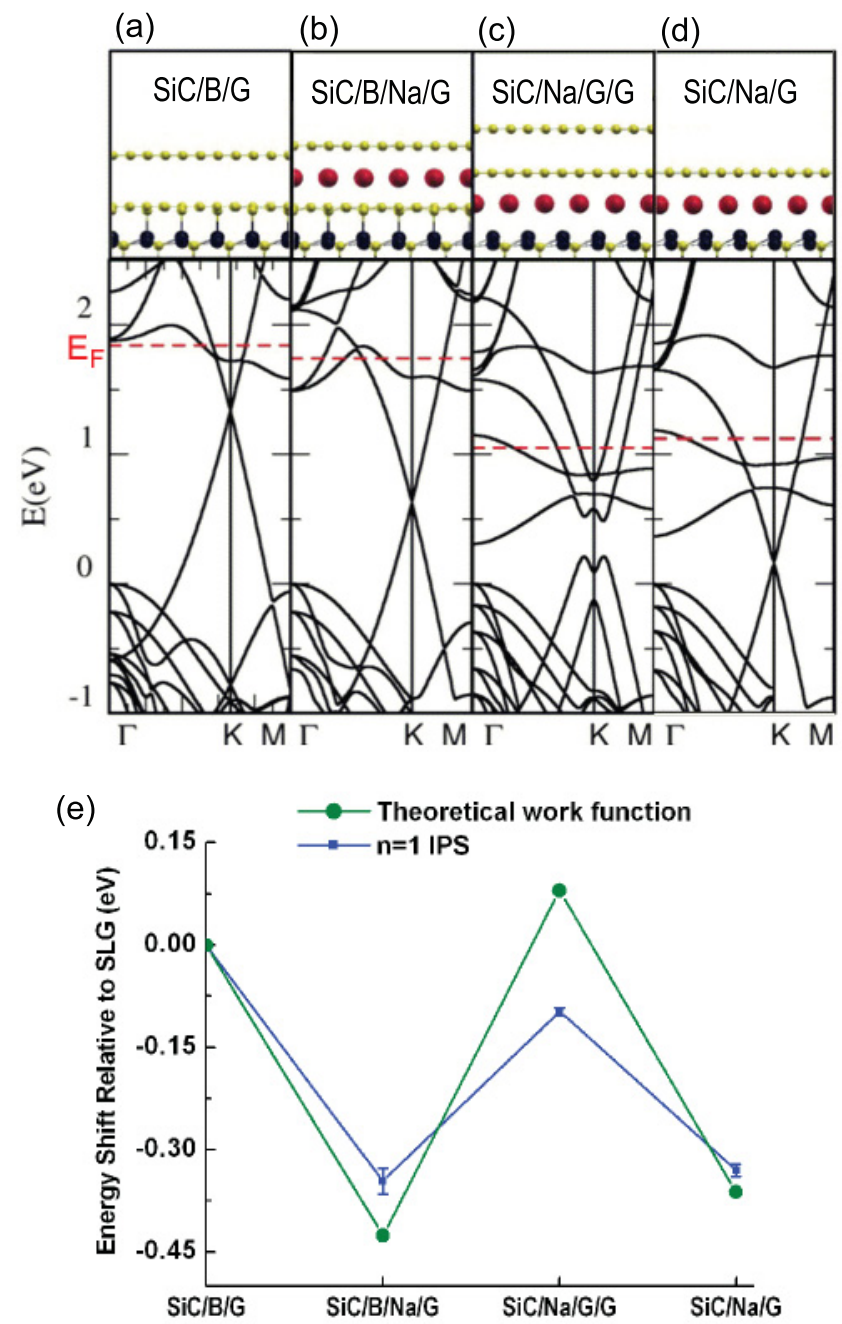

FIG. 4. (Color online) Calculated electronic bands and atomic structures for (a) $\mathrm{SLG}$ on top of $\mathrm{SiC}(0001)$, (b) $\mathrm{SiC} / \mathrm{B} / \mathrm{Na} / \mathrm{G}$, (c) $\mathrm{SiC} / \mathrm{Na} / \mathrm{G} / \mathrm{G}$ (layers are $\mathrm{AB}$ stacked), and (d) $\mathrm{SiC} / \mathrm{Na} / \mathrm{G}$. Yellow atoms are $\mathrm{C}$, black are $\mathrm{Si}$, and red are Na. (e) Shift in measured $n=1$ IPS energy (blue squares) and shift in calculated work function from SLG (green circles) for each intercalation structure.

For $\mathrm{SiC} / \mathrm{B} / \mathrm{Na} / \mathrm{G}$, the $n=1$ state is shifted down (purple curve) compared to bare $\mathrm{SLG}$ on $\mathrm{SiC}(0001)$. This shift can be attributed to a lowering of the work function as sodium electron dopes the graphene through charge transfer. For $\mathrm{SiC} / \mathrm{Na} / \mathrm{G} / \mathrm{G}$, the $n=1$ IPS is shifted up again and is slightly lower in energy compared to SLG (green curve). This reversal in direction of work function can be explained by the hypothesis that $\mathrm{Na}$ atoms penetrate beneath the buffer layer so that their doping of both layers appears reduced compared to when sodium sits between buffer and graphene layer. This is analogous to the differences in substrate-induced doping between single-layer and bilayer epitaxial graphene on $\mathrm{SiC}(0001) .{ }^{31}$ For $\mathrm{SiC} / \mathrm{Na} / \mathrm{G}$ (blue curve), the downshift in $n=1$ IPS implies that electron doping is increased and work function decreased in a similar manner to $\mathrm{SiC} / \mathrm{B} / \mathrm{Na} / \mathrm{G}$. This similarity is expected because both these structures have $\mathrm{Na}$ intercalated directly under one graphene sheet.

Our interpretation of the location of intercalated $\mathrm{Na}$ atoms and associated electron-doping effects of the three different in- tercalation structures can be substantiated using first-principles electronic structure calculations. Figure 4 displays theoretical band structures for SLG [Fig. 4(a)] along with $\mathrm{SiC} / \mathrm{B} / \mathrm{Na} / \mathrm{G}$ [Fig. 4(b)], SiC/Na/G/G [Fig. 4(c)] and SiC/Na/G [Fig. 4(d)]. For SLG, Si dangling bonds at the SiC-buffer layer interface are responsible for a charge transfer, which induces a shift of the Dirac Point to 0.49 eV below the Fermi level (red dashed line in Fig. 4). For $\mathrm{SiC} / \mathrm{B} / \mathrm{Na} / \mathrm{G}$ the Dirac point is shifted down further to $1.09 \mathrm{eV}$ below Fermi level. This is consistent with an earlier calculation made for a similar geometry that first predicted the stability of $\mathrm{SiC} / \mathrm{B} / \mathrm{Na} / \mathrm{G}$ compared to $\mathrm{Na}$ adsorbed on top of graphene. ${ }^{29}$ In addition, the shift is similar in size to the shift of the Dirac point reported for $\mathrm{Ca}$ intercalation by McChesney et al. ${ }^{21}$ It would be of great interest to extend our calculations to consider the possibility of a spin-polarized band structure similar to the calculations for Mn in Ref. 17.

Our DFT calculations for $\mathrm{SiC} / \mathrm{Na} / \mathrm{G} / \mathrm{G}$ shown in Fig. 4(c) prove theoretically that the $\mathrm{Na}$ atoms between the buffer layer and SiC surface decouple the buffer layer. Such a decoupling has been addressed theoretically for the case of $\mathrm{H}$ intercalation beneath epitaxial graphene on $\mathrm{SiC}^{32}$ but not for the case of alkalai metal atoms. This growing body of both theoretical understanding and experimental observations suggests that such decoupling is likely to be a routine feature of intercalation processes on $\mathrm{SiC}$.

The electron doping for the AB-stacked graphene layers that remain after buffer layer decoupling to make $\mathrm{SiC} / \mathrm{Na} / \mathrm{G} / \mathrm{G}$ is reduced compared to $\mathrm{SiC} / \mathrm{B} / \mathrm{Na} / \mathrm{G}$ and a $0.29-\mathrm{eV}$ gap opens around the Dirac point with midgap located $0.65 \mathrm{eV}$ below the Fermi level. For $\mathrm{SiC} / \mathrm{Na} / \mathrm{G}$ [Fig. 4(d)], doping from intercalated $\mathrm{Na}$ results in a downshift of its Dirac point to $0.99 \mathrm{eV}$ below the Fermi level. Importantly, the linear band structure around the Dirac point is maintained in this isolated, activated buffer, indicating that it is a true graphene sheet in accordance with the direct STM imaging in Fig. 3(f).

To compare IPS energy shifts with theory, we used the results of $a b$ initio calculations to extract work functions for each structure. The image in Fig. 4(e) displays IPS $n=1$ position and calculated work-function shift relative to the reference point of SLG. The trend of the calculated work-function changes agrees with the STS measurements of the change in $n=1$ IPS peak position. The most important correspondence from the comparison in Fig. 4(e) is the reversal in direction of the work-function change in going from $\mathrm{SiC} / \mathrm{B} / \mathrm{Na} / \mathrm{G}$ to $\mathrm{SiC} / \mathrm{Na} / \mathrm{G} / \mathrm{G}$ intercalation. This verifies the hypothesis that heating of the sample initiates $\mathrm{Na}$ atom intercalation beneath the buffer layer and not in direct contact with the upper graphene layer.

\section{SUMMARY AND CONCLUSIONS}

In summary, we observed the intercalation of sodium deposited on SLG grown on $\mathrm{SiC}(0001)$. Contrary to graphite, sodium intercalates readily at the surface at room temperature and forms two different structures. At first, the sodium goes in between the top single graphene layer and the carbon buffer layer. Over time, or with annealing, sodium penetrates through the buffer layer converting this into a second graphene layer. Buffer layer decoupling by intercalation is directly observed by deposition of $\mathrm{Na}$ onto bare buffer layer followed by annealing. 
First-principles DFT calculations directly show the electronic structure and work-function trends for these structures. The latter compare favorably with measured shifts in IPS energies. These observations point out the rich possibilities for tailoring electronic structure by intercalation of graphene. The very strong electron doping reported here for $\mathrm{Na}$ intercalation could be valuable in the further exploration of superconductivity and magnetism in graphene. ${ }^{21}$ Moreover, the efficiency of the intercalation processes identified for $\mathrm{Na}$ suggests that intercalation of epitaxial graphene may be an even more versatile strategy for functional modification than it is for graphite because $\mathrm{Na}$ does not intercalate graphite. Importantly, the diversity of structures that we have observed as well as their coexistence with one another must be carefully considered in order to correctly interpret spatially averaging experiments on intercalated graphene.

\section{ACKNOWLEDGEMENT}

This work was funded by the National Science Foundation Center for Chemical Innovation: Center for Molecular Spintronics under Grant No. CHE-0943975.
*Present address: Department of Physics and Department of Chemistry, University of North Texas, Denton, TX 76203.

†dbdoughe@ncsu.edu

${ }^{1}$ A. K. Geim and K. S. Novoselov, Nat. Mater. 6, 183 (2007).

${ }^{2}$ Y.-M. Lin, C. Dimitrakopoulos, K. A. Jenkins, D. B. Farmer, H.-Y. Chiu, A. Grill, and P. Avouris, Science 327, 662 (2010).

${ }^{3}$ M. S. Dresselhaus and G. Dresselhaus, Adv. Phys. 51, 1 (2002).

${ }^{4}$ T. E. Weller, M. Ellerby, S. S. Saxena, R. P. Smith, and N. T. Skipper, Nat. Phys. 1, 39 (2005).

${ }^{5}$ W. Metz and L. Siemsglüss, Mater. Sci. Eng. 31, 119 (1977).

${ }^{6}$ A. Mattausch and O. Pankratov, Phys. Rev. Lett. 99, 076802 (2007).

${ }^{7}$ K. V. Emtsev, F. Speck, T. Seyller, L. Ley, and J. D. Riley, Phys. Rev. B 77, 155303 (2008).

${ }^{8}$ C. Riedl, C. Coletti, T. Iwasaki, A. A. Zakharov, and U. Starke, Phys. Rev. Lett. 103, 246804 (2009).

${ }^{9}$ C. Virojanadara, A. A. Zakharov, R. Yakimova, and L. I. Johansson, Surf. Sci. 604, L4 (2010).

${ }^{10}$ S. Watcharinyanon, C. Virojanadara, J. R. Osiecki, A. A. Zakharov, R. Yakimova, R. I. G. Uhrberg, and L. I. Johansson, Surf. Sci. 605, 1662 (2011).

${ }^{11}$ S. Oida, F. R. McFeely, J. B. Hannon, R. M. Tromp, M. Copel, Z. Chen, Y. Sun, D. B. Farmer, and J. Yurkas, Phys. Rev. B 82, 041411R (2010).

${ }^{12}$ A. L. Walter, K. J. Jeon, A. Bostwick, F. Speck, M. Ostler, T. Seyller, L. Moreschini, Y. S. Kim, Y. J. Chang, K. Horn, and E. Rotenberg, Appl. Phys. Lett. 98, 184102 (2011).

${ }^{13}$ C. Virojanadara, S. Watcharinyanon, A. A. Zakharov, and L. I. Johansson, Phys. Rev. B 82, 205402 (2010).

${ }^{14}$ C. Virojanadara, A. A. Zakharov, S. Watcharinyanon, R. Yakimova, and L. I. Johansson, New J. Phys. 12, 125015 (2010).

${ }^{15}$ Y. C. Cheng and U. Schwingenschlogl, Appl. Phys. Lett. 97, 193304 (2010).

${ }^{16}$ B. Premlal, M. Cranney, F. Vonau, D. Aubel, D. Casterman, M. M. De Souza, and L. Simon, Appl. Phys. Lett. 94, 263115 (2009).
${ }^{17}$ T. Jayasekera, B. D. Kong, K. W. Kim, and M. Buongiorno Nardelli, Phys. Rev. Lett. 104, 146801 (2010).

${ }^{18}$ A. Bostwick, T. Ohta, T. Seyller, K. Horn, and E. Rotenberg, Nat. Phys. 3, 36 (2007).

${ }^{19}$ A. Bostwick, F. Speck, T. Seyller, K. Horn, M. Polini, R. Asgari, A. H. MacDonald, and E. Rotenberg, Science 328, 999 (2010).

${ }^{20}$ T. Ohta, A. Bostwick, T. Seyller, K. Horn, and E. Rotenberg, Science 313, 951 (2006).

${ }^{21}$ J. L. McChesney, A. Bostwick, T. Ohta, T. Seyller, K. Horn, J. Gonzalez, and E. Rotenberg, Phys. Rev. Lett. 104, 136803 (2010).

${ }^{22}$ P. M. Echenique and J. B. Pendry, J. Phys. C 11, 2065 (1978).

${ }^{23}$ S. Bose, V. M. Silkin, R. Ohmann, I. Brihuega, L. Vitali, C. H. Michaelis, P. Mallet, J. Y. Veuillen, M. A. Schneider, E. V. Chulkov, P. M. Echenique, and K. Kern, New J. Phys. 12, 023028 (2010).

${ }^{24}$ V. M. Silkin, J. Zhao, F. Guinea, E. V. Chulkov, P. M. Echenique, and H. Petek, Phys. Rev. B 80, 121408R (2009).

${ }^{25}$ A. Sandin, A. Pronschinske, J. E. Rowe, and D. B. Dougherty, Appl. Phys. Lett. 97, 113104 (2010).

${ }^{26}$ D. B. Dougherty, P. Maksymovych, J. Lee, and J. T. Yates, Phys. Rev. Lett. 97, 236806 (2006).

${ }^{27}$ Alex Pronschinske, Daniel J. Mardit, and Daniel B. Dougherty, Phys. Rev. B 84, 205427 (2011).

${ }^{28}$ P. Giannozzi, S. Baroni, N. Bonini, M. Calandra, R. Car, C. Cavazzoni, D. Ceresoli, G. L. Chiarotti, M. Cococcioni, I. Dabo, A. Dal Corso, S. de Gironcoli, S. Fabris, G. Fratesi, R. Gebauer, U. Gerstmann, C. Gougoussis, A. Kokalj, M. Lazzeri, L. Martin-Samos, N. Marzari, F. Mauri, R. Mazzarello, S. Paolini, A. Pasquarello, L. Paulatto, C. Sbraccia, S. Scandolo, G. Sclauzero, A. P. Seitsonen, A. Smogunov, P. Umari, and R. M. Wentzcovitch, J. Phys. Condens. Matter 21, 395502 (2009).

${ }^{29}$ S. M. Choi and S. H. Jhi, Appl. Phys. Lett. 94, 153108 (2009).

${ }^{30}$ M. T. Johnson, H. I. Starnberg, and H. P. Hughes, Surf. Sci. 178, 290 (1986).

${ }^{31}$ T. Filleter, K. V. Emtsev, T. Seyller, and R. Bennewitz, Appl. Phys. Lett. 93, 113117 (2008).

${ }^{32}$ B. Lee, S. Han, and Y.-S. Kim, Phys. Rev. B 81, 075432 (2010). 